\title{
A Bayesian 3D Volume Reconstruction for Confocal Micro-rotation Cell Imaging
}

\author{
Yong Yu, Alain Trouvé, and Bernard Chalemond \\ Ecole Normale Supérieure de Cachan, France \\ $\left\{\right.$ yu, trouve, Bernard. chalmond $\varrho_{\mathrm{cmla}}$.ens-cachan. fr
}

\begin{abstract}
Recently, micro-rotation confocal microscopy has enabled the acquisition of a sequence of slices for a non-adherent living cells where the slices' positions are roughly controlled by a dielectric-field biological cage. The high resolution volume reconstruction requires then the integration of precise alignment of slice positions. We propose in the Bayesian context, a new method combining both slice positioning and 3D volume reconstruction simultaneously, which leads naturally to an energy minimization procedure of a variational problem. An automatic calibration paradigm via Maximum Likelihood estimation (MLE) principle is used for the relative hyper-parameter determination. We provide finally experimental comparison results on both conventional z-stack confocal images and 3D volume reconstruction from micro-rotation slices of the same non-adherent living cell to show its potential biomedical application.
\end{abstract}

\section{Introduction}

Recently, thanks to the combining efforts of both biological and physical research, it emerges a novel specification and design methodology [5][8] for manipulating microscopic objects by a dielectric-field micro-rotation cage. One of its immediate impact is its fruitful application to non-adherent living cell imaging without sticking to a glass capillary [2]. Although there already exists axial tomographic confocal microscopy techniques [4] improving the imaging resolution by physically rotating the objects, the inherent defocus aberration of conventional z-stack imaging is not yet avoided. As a result, a sophisticated deconvolution process with depth-dependant point spread function is needed to remove optical artifacts. The micro-rotation cage yields continuous rotation movement of a captured object while the focal plane position of the confocal microscopy is fixed (there is no z-direction displacement). In such a way, a sequence of high resolution and isotropic (the point spread function is constant for each slice) 2D optical cross-section images called slices is obtained.

The arising challenge of this new imaging system is to determine precisely the position of each slice before we can reconstruct a high resolution 3D fluorescence intensity volume. Indeed, its novelty comes from coupling two problems which are intensively surveyed in medical imaging processing domain: If these positions were known, the problem would be similar to the classical interpolation problem in the simplest case, and in more complicated cases to the deconvolution or tomography problems [6]. On the other hand, if the 3-D intensity volume is known, the estimation of the positions of a particular slice, would also reduce to the classical problem of registration [124]. So, we 
need to integrate these two sub-problems into a common formulation which implies performing simultaneously registration (slice positioning in our case) and reconstruction.

As a first step, being aware of the computational overhead introduced by any deconvolution procedure for the sequence of micro-rotation slices, we have, for the moment, emphasized the estimation of slices' geometry parameters without taking the PSF into account in the imaging process.

The reconstructed volume is modeled as a Gaussian process [7] to characterize the spatial coherence between slices. As a result, cell reconstruction including parameters estimation and slice positioning is performed in a statistical framework from which derives naturally a variational formulation. We will show in the experiment section that our simplified volume model does not degrade its high resolution reconstruction comparing with the conventional z-stack result on a real cell example.

The paper is organised as following: In section 2, a MLE parameter calibration paradigm is proposed to tune automatically the hyper-parameters of the statistical modelling. In section 3, the variational formulation is derived from Bayesian inference. Moreover, The Fast Gaussian Transform (FGT) method is shortly summarized for the sake of its role in our numeric solution. Finally, in section 4, the visualization experiment on real non-adherent living cell is demonstrated with the comparison to conventional z-stack images.

\section{Statistical Modelling and MLE Parameter Calibration}

We fix now the notations. We denote $\left(I_{i}\right)_{1 \leq i \leq N}$ the sequence of $N$ image slices. The slice positions are coded by $N$ rigid transformations $\Phi \doteq\left(\varphi_{i}\right)_{1 \leq i \leq N}=\left(R_{i}, b_{i}\right)_{1 \leq i \leq N}$ which include pairs of rotation matrix and translation vector acting on a reference plane $H_{0}$ after choosing a space frame. We denote $f$, the continuous intensity $3 \mathrm{D}$ volume to be reconstructed.

In our statistical model, the volume $f$ is modeled as a centered 1 Gaussian field with covariance a translation and rotation invariant covariance function $k(.,$.$) . For the sake$ of simplicity, we choose a Gaussian kernel so that $k(x, y)=\sigma_{f}^{2} \exp \left(-\|y-x\|^{2} /\left(2 \lambda_{f}^{2}\right)\right)$ where $\lambda_{f}$ plays the role of a scale parameter and $\sigma_{f}^{2}$ the variance of the induced stationary process.

Any observed slice $I_{i}$ is modeled, given the 3D positioning $\phi_{i}$, as a noisy version of the restriction $f\left(\varphi_{i}\left(x_{s}\right)\right)_{x_{s} \in H_{0}}$, i.e.,

$$
I_{i}\left(x_{s}\right)=f\left(\phi_{i}\left(x_{s}\right)\right)+\sigma_{\epsilon} \epsilon_{i, s} .
$$

where the $\epsilon_{i, s}$ is defined as a Gaussian white noise. Since $f \circ \phi$ and $f$ for $\phi$ fixed have the same distribution as a Gaussian process, we deduce easily that $I_{i} \sim \mathcal{N}(0, \Gamma)$ with

$$
\Gamma\left(x_{s}, x_{t}\right)=\sigma_{f}^{2} e^{\left(-\frac{\left\|x_{s}-x_{t}\right\|^{2}}{2 \lambda_{f}^{2}}\right)}+\sigma_{\epsilon}^{2} \mathbf{1}_{s=t}
$$

\footnotetext{
${ }^{1}$ It is not a strong assumption since the constant gray level of background is easily measured and then subtracted.
} 
Let $\theta=\left(\tilde{\sigma}_{f}^{2}, \lambda_{f}, \sigma_{\epsilon}^{2}\right)$ where $\tilde{\sigma}_{f}^{2}=\sigma_{f}^{2} / \sigma_{\epsilon}^{2}$. We have $\Gamma\left(x_{s}, x_{t}\right)=\sigma_{\epsilon}^{2} \tilde{\Gamma}\left(x_{s}, x_{t}\right)$ with $\tilde{\Gamma}\left(x_{s}, x_{t}\right)=\tilde{\sigma}_{f}^{2} \exp \left(-\frac{\left\|x_{s}-x_{t}\right\|^{2}}{2 \lambda_{f}^{2}}\right)+\mathbf{1}_{s=t}$.

Making the simplifying assumption of the conditional independence the slices $I_{i}$ 's given $\Phi$, the log-likelihood of the whole sequence of slices is:

$$
\log P(I \mid \theta, \Phi)=\sum_{i=1}^{N} \log P\left(I_{i} \mid \theta\right)=-\sum_{i=1}^{N}\left(\frac{1}{2 \sigma_{\epsilon}^{2}} I_{i}^{\prime} \tilde{\Gamma}^{-1} I_{i}+\frac{1}{2} \log \left(\left|\sigma_{\epsilon}^{2} \tilde{\Gamma}\right|\right)\right)+C t e
$$

where $I \doteq\left(I_{i}(s)\right)_{1 \leq i \leq N, s \in H_{0}}$. and $C t e$ is the constant factor. The MLE estimation $\hat{\theta}$ of $\theta$ requires maximizing the term $\log P(I \mid \theta)$. Note the optimisation on $\sigma_{\epsilon}^{2}$ is straightforward and gives

$$
\hat{\sigma}_{\epsilon}^{2}=\frac{1}{M N} \sum_{i=1}^{N} I_{i}^{\prime} \tilde{\Gamma}^{-1} I_{i}
$$

where $M$ is the number of pixels of each slice so that the MLE can be reduced to the optimisation of the two parameters $\left(\tilde{\sigma}_{f}^{2}, \lambda_{f}\right)$ done by exhaustive search on a grid. To save computation, we perform the estimation on a family of sub-regions of moderate sizes so that the inversion of the $\tilde{\Gamma}$ 's are easily feasible.

\section{MAP Estimation}

We recover $(f, \Phi)$ given $I$ by maximum a posteriori estimation (MAP). We assume that $f$ and $\Phi$ are independent. The distribution of $f$ has been defined before so that we need to precise the prior distribution for $\Phi$.

Since it is relatively easy to determine both the mean axis orientation and the angular speed of the micro-rotation movement, we start from an ideal movement trajectory $\Phi_{0}$ and $\Phi$ is modelled as a random perturbation of $\Phi_{0}$. More precisely,

$$
\begin{aligned}
P_{\Phi^{0}}(\Phi) & =\prod_{i=1}^{N} P_{\varphi_{i}^{0}}\left(\varphi_{i}\right) \\
P_{\varphi_{i}^{0}}\left(\varphi_{i}\right) & \propto \exp \left(-d^{2}\left(\varphi_{i}, \varphi_{i}^{0}\right)\right)
\end{aligned}
$$

where

$$
d^{2}\left(\varphi_{i}, \varphi_{i}^{0}\right)=\frac{d^{2}\left(R_{i}, R_{i}^{0}\right)}{\sigma_{\omega}^{2}}+\frac{d^{2}\left(b_{i}, b_{i}^{0}\right)}{\sigma_{b}^{2}}
$$

and two variance parameter $\sigma_{\omega}^{2}$ and $\sigma_{b}^{2}$ described the perturbation strength.

The distance between two rotation matrices $R_{1}$ and $R_{2}$ in 3D space is defined as the common geodesic distance which is invariant to right/left rotation multiplication:

$$
d\left(R_{1}, R_{2}\right)=\cos ^{-1}\left(\frac{\operatorname{trace}\left(R_{1} R_{2}^{-1}\right)-1}{2}\right)
$$


Moreover, the distance between two translations $b_{1}$ and $b_{2}$ is the common Euclidean distance $d^{2}\left(b_{1}, b_{2}\right)=\left\|b_{1}-b_{2}\right\|_{\mathbb{R}^{3}}^{2}$.

Finally, the MAP estimation gives us an equivalent variational problem:

$$
\begin{aligned}
\mathcal{J}(\Phi, f)= & \frac{1}{2} \sum_{i=1}^{N} d^{2}\left(\varphi_{i}, \varphi_{i}^{0}\right)+\frac{1}{2}\|f\|_{\mathcal{H}}^{2} \\
& +\frac{1}{2} \sum_{i=1}^{N} \sum_{x \in H_{0}}\left|f\left(\varphi_{i}(x)\right)-I_{i}(x)\right|^{2} / \sigma_{\epsilon}^{2} .
\end{aligned}
$$

where $\mathcal{H}$ is a reproducing kernel Hilbert space (RKHS) associated with covariance function $k$.

\subsection{Gradient Computations}

To minimize $\mathcal{J}$, we use a gradient-descent based method defined as

$$
\left(\begin{array}{c}
\Phi(t+\delta t) \\
f(t+\delta t)
\end{array}\right)=\left(\begin{array}{c}
\Phi(t) \\
f(t)
\end{array}\right)-\left(\begin{array}{c}
\nabla_{\Phi(t)} \mathcal{J} \\
\nabla_{f(t)} \mathcal{J}
\end{array}\right) \delta t
$$

As known with RKHS (see [9]), one can introduce a finite family $\left(x_{i}^{c}\right)_{1 \leq i \leq N_{C}}$ of control points defined on a grid and approximate $f \in \mathcal{H}$ by projection as a linear combination

$$
f(x)=\sum_{x_{i}^{c}} \alpha_{i} k\left(x, x_{i}^{c}\right)
$$

Now the differential of $\nabla_{f} \mathcal{J}$ is reduced to a finite dimensional expression $\nabla_{\alpha} \mathcal{J}$ due to Equ.9.

$$
\nabla \alpha \mathcal{J}=K \alpha+A^{T}(A \alpha-I) / \sigma_{\epsilon}^{2},
$$

where

$$
\begin{gathered}
K \doteq\left(k\left(x_{i}^{c}, x_{j}^{c}\right)\right)_{1 \leq i, j \leq N_{C}} \\
A \doteq\left(k\left(\varphi_{i}(s), x_{j}^{c}\right)\right)_{1 \leq i \leq N, s \in H_{0}, 1 \leq j \leq N_{C}}
\end{gathered}
$$

We decompose the partial gradient $\nabla_{\Phi} \mathcal{J}$ into the two partial gradients: $\left(\nabla_{\mathbf{R}_{\mathbf{i}}} \mathcal{J}\right)_{1 \leq i \leq N}$ and a translation related term denoted by $\left(\nabla_{\mathbf{b}_{i}} \mathcal{J}\right)_{1 \leq i \leq N}$, which are calculated directly:

$$
\nabla b_{i} \mathcal{J}=\frac{b_{i}-b^{0}}{\sigma_{b}^{2}}+\sum_{x \in H_{0}} \frac{\left(f\left(\varphi_{i}(x)\right)-I_{i}(x)\right)}{\sigma_{\epsilon}^{2}} \nabla f\left(\varphi_{i}(x)\right) .
$$

and

$$
\nabla_{R_{i}} \mathcal{J}=\frac{1}{2} \sum_{j=1}^{3}\left(R_{i}\right)_{. j} \wedge\left(\frac{\partial \mathcal{J}}{\partial R_{i}}\right)_{. j} \wedge R_{i}
$$

where $\frac{\partial \mathcal{J}}{\partial R_{i}}$ is coefficient-wise differential of $\mathcal{J}$ with respect to the $R_{i}$ 's coefficients and operator ()$_{. j}$ extracts the matrix's $j^{\text {th }}$ column and $\wedge$ is the common cross product. 


\subsection{Fast Gaussian Transform}

Since the computation of $\nabla_{\alpha} \mathcal{J}$ and $\nabla_{\Phi} \mathcal{J}$ involved intensive evaluation of $f(x)$ in Equ. 9, a brute force implementation is hopeless for the volume reconstruction. We have used the recently popularized Fast Gaussian Transform method improved by C. Yang et al [10] which exploits the fast decay of the Gaussian kernel based on the work of Greengard and Strain [3]. We mention only its basic principle while more implementation details can be found in their original paper [10].

As all multipole methods, FGT method expands the evaluation function around a chosen pole $\bar{x}$ into two separate terms:

$$
\begin{aligned}
f(x) & =\sum_{x_{i}} \alpha_{i} e^{-\frac{\left\|x-x_{i}\right\|^{2}}{2}}=e^{-\frac{\|x-\bar{x}\|^{2}}{2}} \sum_{x_{i}} \alpha_{i} e^{-\frac{\left\|x_{i}-\bar{x}\right\|^{2}}{2}} e^{<x-\bar{x}, x_{i}-\bar{x}>} \\
& \approx \sum_{0 \leq \sum a_{i} \leq p} \frac{1}{a !} e^{-\frac{\|x-\bar{x}\|^{2}}{2}}(x-\bar{x})^{a} \underbrace{\sum_{x_{i}} \alpha_{i} e^{-\frac{\left\|x_{i}-\bar{x}\right\|^{2}}{2}}\left(x_{i}-\bar{x}\right)^{a}}_{\text {term independent of } x}
\end{aligned}
$$

where we denote the polynomial exponent degrees $a \doteq\left(a_{1}, \cdots, a_{d}\right), a ! \doteq \prod_{i=1}^{d} a_{i}$, $x^{a} \doteq \prod_{i=1}^{d} x_{i}^{a_{i}}(\mathrm{~d}=3$ for $3 \mathrm{D}$ points $)$ and $p$ the polynomial approximation order $(p=6$ in our case). Using the FTG approach, the complexity of the computation of a product $K \alpha$ is reduced from $O\left(N_{C}^{2}\right)$ to $O\left(N_{C}\right)$.

\section{Experimental Results}

We have performed the experiment on the image sequences from both simulation data and real imaging data. But to save the space we don't report here the result from the simulation data and focus only on that from the real imaging data.

The real imaging data shown in Fig. 1 (sampling from one tour of 340 slices) was acquired by our biologist collaborators from a sw13/20 living cell caged and suspended in a Cytocon ${ }^{\mathrm{TM}}$ chip (Evotec technologies, Germany) to investigate the localization and dynamics of nuclear lamina and green fluorescent protein (GFP). These confocal images were then collected using a Zeiss Axiovert ${ }^{\text {TM }} 200$ typed confocal microscopy. For the optical parameter setting, a 63x water immersion objective is used and numerical aperture (NA) is set to 1.2 . Finally, the resolution of each optical section image is $129 \mathrm{~nm}$ and the chip driver gives us the mean rotation direction projected in $2 \mathrm{D}$ optical section (it is y axis or vertical direction in this case study).

Before launching the reconstruction-alignment coupling processing, the parameters of $\theta$ needed for the variational formula are estimated by the method proposed in section 2 on 100 blocks of $30 \times 30$ uniformly distributed in all 340 slices (the size of each slice is $156 \times 156$ ). The parameters are estimated by MLE criterion then as $\sigma_{f}^{2}=$ $9.75 \times 10^{6}, \sigma_{\epsilon}^{2}=3.36 \times 10^{5}$ and $\lambda_{f}=3.5$. The remaining two variance parameters coding the instability of the movement away from the ideal trajectory determined by the mean rotation movement are set as $\sigma_{\omega}^{2}=10.0$ and $\sigma_{b}^{2}=10.0$.

Then we have run the optimisation procedure determined by Equ. 8 for 5 iterations. Each iteration contains a subroutine of volume reconstruction driven by conjugate gradient method with fixed 20 iterations and a subroutine of slices alignment driven by 


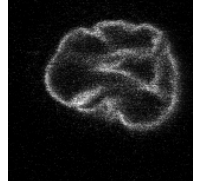

(a)

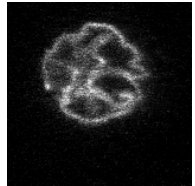

(g)

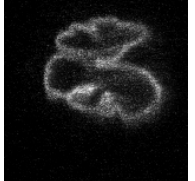

(b)

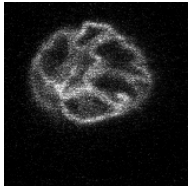

(h)

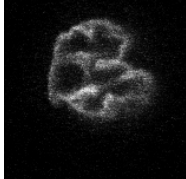

(c)

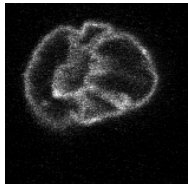

(i)

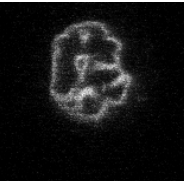

(d)

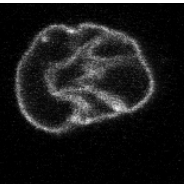

(j)

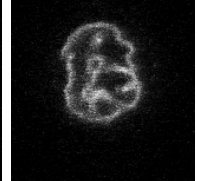

(e)

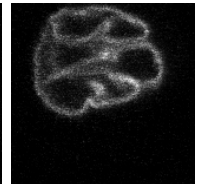

(k)

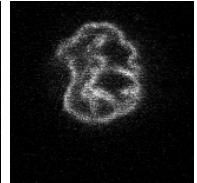

(f)

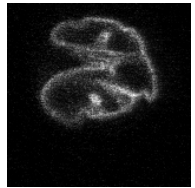

(1)

Fig. 1. 12 micro-rotation slices from a real confocal microscopy imaging data sequence

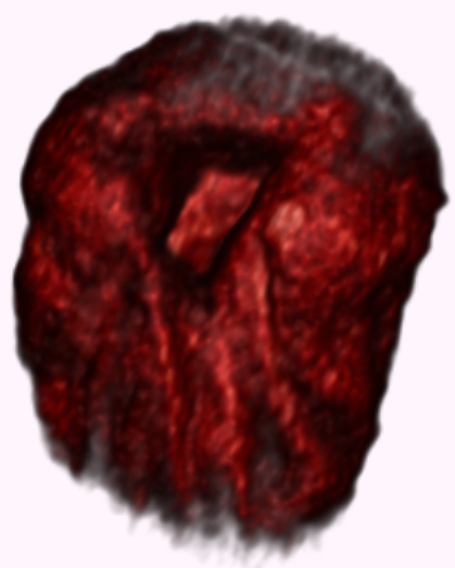

(a)

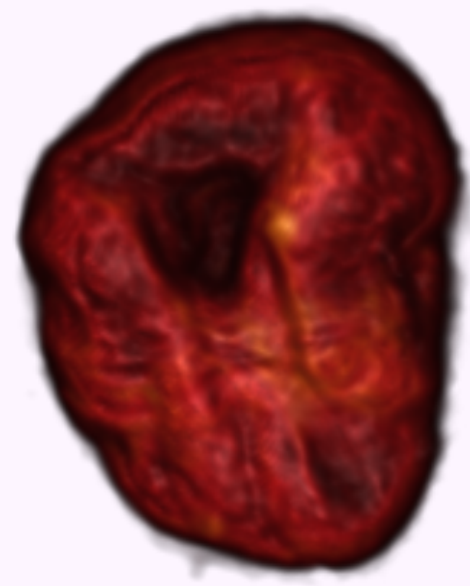

(b)

Fig. 2. A 3D volume rendering case using OsiriX software projected on a same viewing position. (a) From conventional Z stacks already deconvoluted by SVI Huygens deconvolution software. (b) From the reconstruction result of micro-rotation slices.

Levenberg-Marquardt method with 20 - 200 iterations which depends on the distance between the initial and final values of slices positions.

In order to have a fair validation of the reconstruction based on micro-rotation data, we provide also a reconstruction based on the state-of-art $\mathrm{Z}$-stack imaging techniques. The Z-stack data have acquired in the suspension mode of the Cytocon ${ }^{\mathrm{TM}}$ chip now controlled by a piezo motor to displace the whole cage. The step between two planes along $\mathrm{z}$ direction is set to $100 \mathrm{~nm}$ and 181 slices were obtained for the same living cell done as done in micro-rotation mode. 


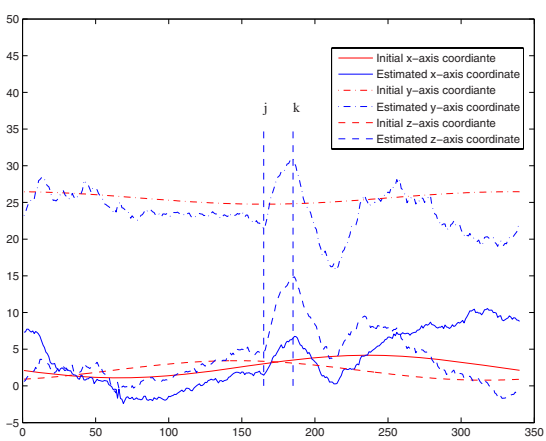

(a)

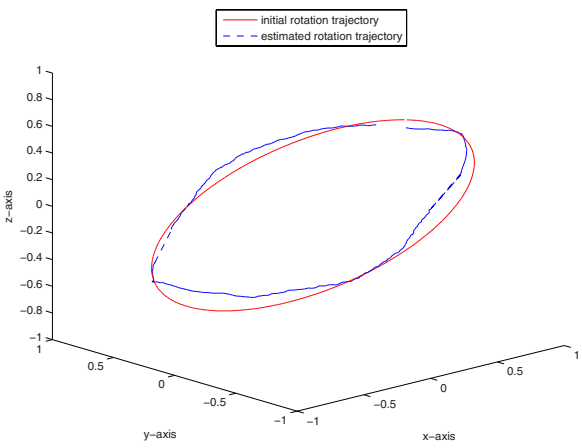

(b)

Fig. 3. Rigid transformation estimation of the 340 micro-rotation slices position parameters: (a) translation of each slice (b) trajectory generated from the 340 rotation matrices given by each slice position acting on a unit vector $\left[\begin{array}{lll}1 & 0 & 0\end{array}\right]^{t}$

The final volume reconstruction result are rendered in the same viewing direction as that the $\mathrm{z}$-stack data (its volume size is $109 \times 109 \times 181$ ) which is shown in Fig. 2. The positions of each slice coded by rigid transformation parameters are shown in Fig. 3 . which represents an irregular perturbations in agreement with physical models.

This irregular perturbation is apparent on Fig. 1, in particular from instant (j) to (k) (upward jump). Note that this jump is well detected on the position parameters in Fig 3. The deeper biological evaluation is beyond the scope of this paper. However, it is clear from the rendering volume viewing shown in Fig. 2 that the reconstruction quality from micro-rotation slices has been improved more than that from deconvoluted z-stack slices: not only it gives the cellular membrane which is missing in the $\mathrm{z}$-stack volume, but also the geometry distorsion caused by aberration effect has been diminished.

\section{Conclusion}

In this paper we have proposed for a novel micro-rotation confocal microscopy imaging system, a high resolution 3D volume reconstruction method with the simultaneous alignment of each rotational slices. The parameter calibration is performed from a statistical framework via MLE principle. The slices' relative positions are well aligned so that the reconstruction gives more detail than that from the conventional z-stack volume even without the deconvolution refinement.

The immediate on-going improvement work is adopting the multi-resolution strategy by replacing the optimal mono-scale kernel with a combination of kernels at different scale spaces. Not only the releasing of computational burden, but also its ability to avoid local minima during the optimization phase benefit from the multi-resolution strategy. 


\section{Acknowledgements}

This research is supported by the European Commission (NEST 2005 Programme) in consortium AUTOMATION, coordinated by S.L. Shorte (Institut Pasteur, http://www. pfid.org/AUTO MATION/ home/) and by the French Ministry of Research (grant ACINIM FLUTOMY 2003 and Postdoctoral Fellowship 2004). We thank FPID of Institut Pasteur for the supply of the micro-rotation images.

\section{References}

1. Baheerathan, S., Albregtsen, F., Danielsen, H.E.: Registration of serial sections of mouse liver cell nuclei. Journal of Microscopy 192(1), 37-53 (1998)

2. Bradl, J., Rinke, B., Schneider, B., Edelman, P., Hausmann, M., Cremer, C.: Resolution improvement in 3-d microscopy by object tilting. Microsc. Anal. 44, 9-11 (1996)

3. Greengard, L., Strain, J.: A fast algorithm for the evaluation of heat potentials. Comm. Pure Appl. Math. (43), 949-963 (1990)

4. Heintzmann, R., Cremer, C.: Axial tomographic confocal fluorescence microscopy. Journal of Microscopy 206, 7-23 (2002)

5. Shorte, S.L., Muller, T., Schnelle, T.: Method and device for three dimnesional imaging of suspended micro-objects providing for high-resolution microscopy, European patent, No. 1 413911 B1 (2002)

6. Pawley, J.B. (ed.): Handbook of Biological Confocal Microscopy. Springer, Heidelberg (2006)

7. Rasmussen, C.E., Williams, C.: Gaussian Processes for Machine Learning. The MIT Press, Cambridge (2005)

8. Schnelle, T., Hagedorm, R., Fuhr, G., Fielder, S., Muller, T.: Three-dimensional electric field traps for manipulation of cells - calculation and experimental verification. Biochemica et Biophysica Acta 1157, 127-140 (1993)

9. Wahba, G.: Advances in Kernel Methods, chapter Support vector machines, reproducing kernel Hilbert spaces and the randomized GACV, pp. 69-88. MIT Press, Cambridge (1999)

10. Yang, C., Duraiswami, R., Gumerov, N., Davis, L.: Improved fast gauss transform and efficient kernel density estimation. In: IEEE ICCV, pp. 464-471. IEEE Computer Society Press, Los Alamitos (2003) 